\title{
Human monoclonal antibodies in the clinic: novel insights into the choice of specific targets
}

The clinical use of therapeutic antibodies made a great stride forward in 1975 when Köhler and Milstein introduced the hybridoma method [1]. This procedure enabled the production of unlimited amounts of antibodies with predetermined specificity, but it was not applicable to human B cells. Although monoclonal antibodies derived from deliberate immunization of mice, rats and rabbits were not appropriate for the clinic, humanization of these antibodies rendered them pertinent. Thus, a variety of methods was developed to produce monoclonal antibodies for the clinic, even if they were originally fashioned as mouse and rat monoclonal antibodies. Indeed, chimeric and humanized antibodies against cytokines, pathogens and assumed tumor antigens have been approved for therapy.

The alternative means for creating human monoclonal antibodies is the Epstein-Barr virus (EBV) method [2]. This technique, which was the first to generate human monoclonal antibodies, signified a new approach. Its objective was to produce monoclonal antibodies mirroring the human antibody repertoire, presumably reflecting those immune and natural (innate) antibodies that in vivo successfully cope with targets such as pathogens, toxins, tumor cells. Later, this very same concept was extended to the production of human monoclonal antibodies by applying molecular methods in conjunction with single B cell methodologies. Accordingly, antibodies against a variety of antigens (such as bacteria, viruses, toxins, auto-antigens, human blood group antigens and amyloid-beta) were developed from healthy immune individuals.

Obviously, human monoclonal antibodies that neutralize viruses can be used for passive immunization of infected patients. In addition, they can serve to define the exact relevant immunogenic molecular moieties, molecules which resemble those which provide protection. Exact definition of such molecules, as shown for the Dengue virus, provides essential information for the preparation of protective anti-virus vaccines.

Numerous studies in animal models and in humans showed that the cellular immune response was responsible for the rejection of cancer cells. Still, many attempts have been made to develop anti-cancer therapies in the form of monoclonal antibodies to eliminate tumor cells.

Antibodies that target receptors expressed on the surface of target cells can be classified on the basis of their primary mechanism: antagonist antibodies, which block the interaction between receptors and ligands and thus inhibit cell activation, and agonist antibodies which induce receptor-mediated signaling. Recently, a dramatic change took place in the field of immunotherapy, particularly in the field of tumor immunotherapy, related to the type of target chosen for therapeutic monoclonal antibodies. This strategy is based on the cytotoxic potential of lymphoid cells, combined with the use of monoclonal antibodies. Accordingly, antibodies that target lymphocyte surface-specific receptors able to potentiate and unmask the arrested cell's cytotoxic capacity, defined as agonist antibodies, are being developed. Indeed, today pharmaceutical companies are placing much effort on the creation of such antibodies against co-inhibitory cell surface receptors to enhance $\mathrm{T}$ and NK cell activities.

There is a continuous increase in the number of identified receptors expressed on lymphoid cells which mediate the interaction of lymphoid cells with their target cells. At present, several cell-surface molecules 
known to be involved in signaling are already exploited as targets for therapy. Signaling receptors involved in the inhibition of cell reactivity attract special attention with regard to anti-tumor therapy. Antibodies against some of these receptors are surveyed in this special issue of Human Antibodies. Human monoclonal antibodies that target signaling molecules, such as CTLA-4/B7, PD-1/PDL-1, CEACAM-1, TIGIT and DNAM-1, are novel, promising means of coping with cancer cells. Co-stimulatory tumor necrosis factor receptors (TNFR's) can affect the responsiveness of $\mathrm{T}$ cells in recognizing tumor-associated antigens. In fact, human monoclonal agonist antibodies that target TNFR's, such as CD137, CD357, CD134 and CD27, are intriguing future candidates for cancer patient therapy. Additionally, CD38, another receptor expressed on a variety of cells, is involved in anti-tumor activity. Selected agonistic monoclonal antibodies directed against CD38 do increase the expression of that cellsurface molecule, thereby facilitating amplified activation of monocytes and lymphoid cells.

The development of chimeric antigen receptor (CAR) molecular constructs to arm autologous T-cells against target tumor antigens is an additional and promising clinical venue for human monoclonal agonist antibodies. This strategy combines an anti-tumor specific fragment of antibody and activation of the cytotoxic potential of $\mathrm{T}$ cells through intracellular activation motifs. The CAR construct with its scFab is a unique form of an agonist antibody that activates the carrier T-cell upon binding to the target. However, the CAR approach suffers from its dependence on the unrelated and challenging prior assignment to produce xenogeneic monoclonal antibodies against tumorspecific antigens.

In recent years a variety of immunomodulatory human monoclonal antibodies proved to be competent in the treatment of tumors, both in humans and in murine models. Still, complete regressions and cures are irregular and not foreseeable. Several explanations have been put forth, but in many instances it is not clear why some tumor types and patients respond to that treatment, whereas in others it is ineffective. Thus, it is essential to further study the role and mechanisms of anti-signaling cell-surface receptor agonistic antibodies, alone and in conjunction with a) anti-tumor antagonist antibodies and b) conservative anti-tumor treatments.

There is evidence showing involvement of autoimmune responses in the homeostasis of a variety of neural pathologies, including Alzheimer's disease (AD), experimental autoimmune encephalomyelitis (EAE) and multiple sclerosis (MS). Agonistic antibodies directed against oligodendrocytes and neurons in the CNS were shown to attenuate harmful clinical consequences. The antibodies stimulate remyelization of neuron cells and indirectly affect CNS inflammatory cells. The involvement of $\mathrm{T}$ cells and monocytes, together with some of their cytokines in the pathologies of EAE and MS underlines the fundamental ability agonist antibodies might have to activate these cells and their future use in the immunotherapy of neurological disorders.

Today, there is a growing need and a mounting interest to clarify mechanisms involved in the immunotherapy of Alzheimer's disease. This approach follows the interesting results in murine models of $\mathrm{AD}$ and the notion that anti-amyloid-beta $(\mathrm{A} \beta)$ antibodies are present in the sera of healthy individuals, suggesting their role in homeostasis. Accordingly, a variety of human monoclonal antibodies against $\mathrm{A} \beta$ have been developed for passive immunization of $\mathrm{AD}$ patients. Although the mechanism of action of anti-A $\beta$ antibodies is not fully understood, their agonist effect(s) is most likely attained through signaling processes.

Patients who spontaneously have recovered from cancer, patients suffering from autoimmune diseases, and healthy individuals who display protective immune and natural antibodies against lymphoid cellrelated signaling molecules and against other antigens, might be a source of therapeutic human monoclonal antibodies. Such antibodies, similarly to those obtained by the original EBV method, would mirror the clinically-effective antibody repertoire of naturally immunized individuals, rather than that of artificially immunized mice, rats and rabbits.

$$
\begin{array}{r}
\text { Michael Steinitz } \\
\text { Department of Pathology, The Hebrew } \\
\text { University-Hadassah Medical School, Jerusalem } \\
91120 \text {, Israel } \\
\text { e-mail: michaelst@ekmd.huji.ac.il }
\end{array}
$$

\section{References}

[1] G. Köhler and C. Milstein, Continuous cultures of fused cells secreting antibody of predefined specificity, INature $\mathbf{2 5 6}$ (1975), 495-497.

[2] M. Steinitz, G. Klein, S. Koskimies and O. Mäkelä, EB virusinduced B-lymphocyte cell lines producing specific antibody, INature 269 (1977), 420-422. 